\title{
Stably paused genes revealed through inhibition of transcription initiation by the TFIIH inhibitor triptolide
}

\author{
Fei Chen, ${ }^{1}$ Xin Gao, ${ }^{1}$ and Ali Shilatifard ${ }^{1,2}$ \\ ${ }^{1}$ Stowers Institute for Medical Research, Kansas City, Missouri 64110, USA; ${ }^{2}$ Department of Biochemistry and Molecular \\ Genetics, Feinberg School of Medicine, Northwestern University, Chicago, Illinois 60611, USA
}

\begin{abstract}
Transcription by RNA polymerase II (Pol II) in metazoans is regulated in several steps, including preinitiation complex (PIC) formation, initiation, Pol II escape, productive elongation, cotranscriptional RNA processing, and termination. Genome-wide studies have demonstrated that the phenomenon of promoter-bound Pol II pausing is widespread, especially for genes involved in developmental and stimulus-responsive pathways. However, a mechanistic understanding of the paused Pol II state at promoters is limited. For example, at a global level, it is unclear to what extent the engaged paused Pol II is stably tethered to the promoter or undergoes rapid cycles of initiation and termination. Here we used the small molecule triptolide (TPL), an XPB/TFIIH inhibitor, to block transcriptional initiation and then measured Pol II occupancy by chromatin immunoprecipitation (ChIP) followed by next-generation sequencing (ChIP-seq). This inhibition of initiation enabled us to investigate different states of paused Pol II. Specifically, our global analysis revealed that most genes with paused Pol II, as defined by a pausing index, show significant clearance of Pol II during the period of TPL treatment. Our study further identified a group of genes with unexpectedly stably paused Pol II, with unchanged Pol II occupancy even after $1 \mathrm{~h}$ of inhibition of initiation. This group of genes constitutes a small portion of all paused genes defined by the conventional criterion of pausing index. These findings could pave the way for evaluating the contribution of different elongation/pausing factors on different states of Pol II pausing in developmental and other stimulus-responsive pathways.
\end{abstract}

[Keywords: chromatin; RNA polymerase II; transcription]

Supplemental material is available for this article.

Received May 26, 2014; revised version accepted November 7, 2014.

Numerous studies have revealed that transcription by RNA polymerase II (Pol II) is intricately regulated at multiple steps (Hsin and Manley 2012; Kwak and Lis 2013; Smith and Shilatifard 2013). Pol II is recruited to promoters with general transcription factors (GTFs), and starts transcription initiation by synthesizing $\sim 20$ - to 60-nucleotide (nt) RNAs (Rasmussen and Lis 1993). This engaged Pol II could remain in a paused state, waiting for an activating signal; continue to productive elongation; or terminate directly before progressing into the gene body. Genome-wide analysis of Pol II in Drosophila and mammals has shown that Pol II at most genes accumulates at the $5^{\prime}$ end and collectively is referred to as promoter-proximal pausing (Guenther et al. 2007; Muse et al. 2007; Zeitlinger et al. 2007).

The establishment of Pol II promoter-proximal pausing depends on DRB sensitivity-inducing factor (DSIF) and the negative elongation factor (NELF), which together contribute to inhibition of further elongation (Yamaguchi

Corresponding author: ash@northwestern.edu

Article is online at http://www.genesdev.org/cgi/doi/10.1101/gad.246173.114. et al. 2013). Release of paused Pol II into productive elongation requires the positive transcription elongation factor $\mathrm{b}(\mathrm{P}-\mathrm{TEFb})$ within its complex, the super elongation complex (SEC), which phosphorylates DSIF, NELF, and Ser2 of the Pol II C-terminal domain (CTD) (Hsin and Manley 2012; Luo et al. 2012a,b).

Promoter-proximal paused Pol II is prevalent in metazoans, particularly at genes related to developmental and environmental pathways (Core and Lis 2008; Adelman and Lis 2012; Smith and Shilatifard 2013). In Drosophila, it has been shown that minimal promoter elements with paused Pol II are sufficient to mediate a synchronous pattern of gene expression during development (Lagha et al. 2013). Recent work indicates that ribonucleases and mRNA decapping factors function globally in the premature termination of short nascent RNAs, suggesting

(C) 2015 Chen et al. This article is distributed exclusively by Cold Spring Harbor Laboratory Press for the first six months after the full-issue publication date (see http://genesdev.cshlp.org/site/misc/terms.xhtml). After six months, it is available under a Creative Commons License (Attribution-NonCommercial 4.0 International), as described at http:// creativecommons.org/licenses/by-nc/4.0/. 
that promoter-proximal Pol II is unstable and undergoes repetitive cycles of initiation and termination (Brannan et al. 2012; Wagschal et al. 2012; Miki and Grosshans 2013). However, short, capped RNA (scRNA) sequencing (scRNA-seq) revealed that the level of the scRNAs at promoters is unaffected by exosome depletion, implying Pol II could stably associate with promoters (Henriques et al. 2013). One of the best-studied examples of the regulation of transcription by paused Pol II is at the Hsp70 gene in Drosophila. An optical strategy using GFP-tagged Pol II and mCherry-LacI bound to an Hsp70 transgene in the giant nuclei of Drosophila salivary glands demonstrated that promoter-proximal paused Pol II can be stable with a half-life of $5 \mathrm{~min}$ (Buckley et al. 2014). Another recent study measured the average half-life of Pol II pausing at genes in mouse embryonic stem cells at 7 min (Jonkers et al. 2014). Furthermore, a study of 13 genes in Drosophila S2 cells found that the residency was highly variable, with half-lives of Pol II at some promoterproximal regions exceeding $15 \mathrm{~min}$ (Henriques et al. 2013).

In this study, we directly characterized the dynamics of promoter-proximal paused Pol II on a genome-wide scale in HCT116 cells. In order to follow the fate of paused Pol II, we blocked further transcription initiation with the small molecule triptolide (TPL). TPL is an XPB/TFIIH inhibitor (Titov et al. 2011) that we used to prevent the generation of newly engaged Pol II during transcription initiation and measure Pol II occupancy by chromatin immunoprecipitation (ChIP) followed by next-generation sequencing (ChIP-seq). We found that most genes with paused Pol II, as defined by having a high ratio of Pol II occupancy at promoters compared with gene bodies, exhibit a significant clearance of Pol II from both promoters and gene bodies in the presence of TPL. Strikingly, a subclass of genes was identified that maintain unchanged occupancy of promoter-proximal paused Pol II during $1 \mathrm{~h}$ of inhibition of initiation. Importantly, this subclass of genes is less dependent on NELF for maintenance of the paused state than other paused genes. Our study uncovers a remarkable diversity among genes with paused Pol II, raising the question of how the dynamics of genes exhibiting varying paused Pol II states are regulated in a global and gene-specific manner.

\section{Results}

Low-dose TPL inhibits transcription without affecting Pol II levels

After Pol II's initial recruitment to promoters by the basal transcription machinery to form the preinitiation complex (PIC), the establishment of transcriptionally engaged Pol II requires the ATP-dependent helicase/translocase activity of the basal transcription factor TFIIH to unwind the dsDNA to expose the template strand /Goodrich and Tjian 1994; Goodrich et al. 1996; Grumberg and Hahn 2013). TPL has been shown to inhibit in vitro transcription by impeding the ATPase activity of XPB (Titov et al. 2011), the helicase/translocase subunit of TFIIH. In order to determine whether the effect of TPL treatment on transcription is through the inhibition of $\mathrm{XPB}$, we performed nascent RNA sequencing (RNA-seq) with XPBdepleted and TPL-treated cells. We compared the fold change of all transcripts and found a strong positive correlation (correlation coefficient $=0.62$ ) between TPL treatment and XPB depletion (Supplemental Fig. 1A,B), indicating that XPB is a major target of TPL in regard to transcriptional regulation.

Treating cells with TPL can result in proteasomedependent degradation of Pol II (Wang et al. 2011; Manzo et al. 2012). To investigate whether we can inhibit XPBdependent transcription without leading to bulk reductions of Pol II, we performed time and dose response experiments, comparing changes in Pol II levels with changes in transcriptional activity. Western blotting with different Pol II antibodies showed that Pol II levels are unchanged within 90 min of TPL treatment at a concentration of $125 \mathrm{nM}$ (Fig. 1A). However, prolonged TPL inhibition can result in a gradual loss of total Pol II levels.

We performed a similar kinetic analysis of transcriptional competency of TPL-treated cells. To this end, we treated serum-starved HCT116 cells with TPL for various times before $30 \mathrm{~min}$ of serum stimulation. Real-time PCR analysis of known serum-inducible genes-EGR1, FOS, and $J U N B$ - showed that TPL treatment times as short as 30 min reduce their induction (Fig. 1B-D). Moreover, for all genes measured, the expression levels decrease more than twofold during the $60 \mathrm{~min}$ of TPL inhibition despite unchanged Pol II levels (Fig. 1A). Therefore, the comparison
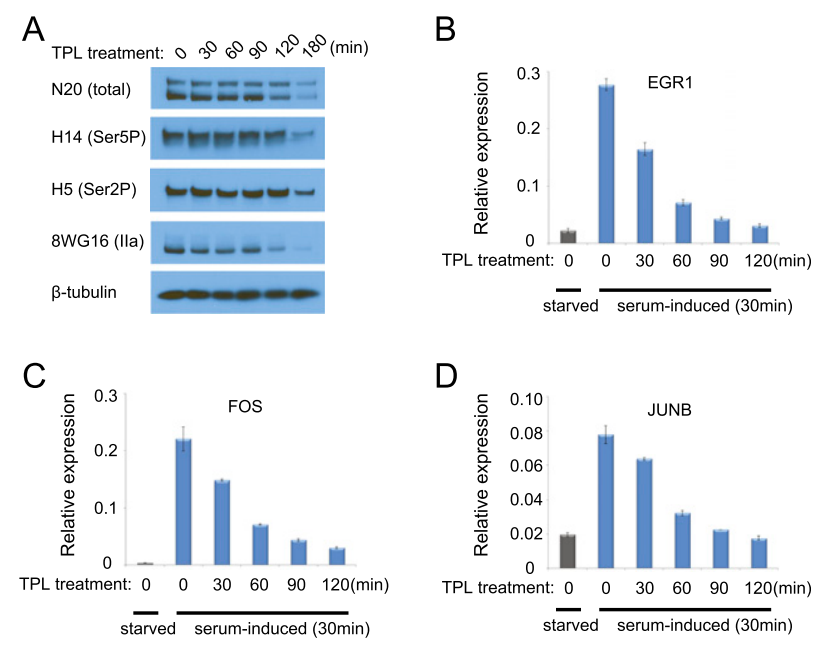

Figure 1. The effect of TPL treatment on Pol II levels and transcriptional activity. (A) Western blot analysis for Pol II in untreated $(0 \mathrm{~min})$ and TPL-treated ( $125 \mathrm{nM})$ HCT 116 cells. Total Pol II (N20), Ser5 phosphorylated Pol II (H14), Ser2 phosphorylated Pol II (H5), and hypophosphorylated Pol II (8WG16) antibodies were used to determine Pol II levels. $(B-D)$ qRTPCR analysis of expression levels of serum-inducible genes EGR1 $(B), F O S(C)$, and JUNB $(D)$ in HCT116 cells. Cells were starved for $48 \mathrm{~h}$ before the addition of serum for $30 \mathrm{~min}$. TPL was added for the indicated time points, except vehicle (DMSO)treated cells (0 min TPL; blue bar) and starved cells (0 min TPL; gray bar). 
between gene expression and bulk Pol II levels indicates that TPL-dependent transcriptional inhibition precedes global Pol II diminution (Fig. 1).

\section{TPL leads to reduced Pol II occupancy on genes and a 5' shift of promoter-proximal Pol II}

Having confirmed that transcription is inhibited within 60 min of TPL treatment without having a major effect on bulk Pol II levels (Fig. 1), we performed ChIP-seq to study Pol II occupancy in the presence and absence of TPL (Fig. 2). HCT116 cells were grown in the absence of serum for $48 \mathrm{~h}$, with TPL or DMSO added after $47 \mathrm{~h}$ of starvation, and serum added back to the cells $24 \mathrm{~min}$ before processing for Pol II ChIP-seq. Metagene analysis reveals a dramatic loss of Pol II both at promoters and in gene bodies (Fig. 2A), indicating a global defect in transcription. The SEC has been shown to be a major regulator of rapid induction of transcription, including for serum-inducible genes (Lin et al. 2011). Western analysis demonstrates that the levels of SEC subunits AFF4 and CDK9 are unchanged during TPL treatment (Supplemental Fig. 2A), suggesting that the degradation of these transcription factors is unlikely to be the cause of the observed changes in Pol II occupancy.

Close inspection of the metagene profiles with and without TPL revealed not only reduced Pol II occupancy but also a shift in the promoter-proximal Pol II in the presence of TPL (Fig. 2B). The shift in the average distribution is $\sim 40 \mathrm{nt}$ toward $5^{\prime}$ in the presence of TPL compared with the DMSO-treated cells. Permanganate footprinting as well as analysis of small RNAs have revealed that Pol II transcribes $\sim 20-60 \mathrm{nt}$ from the transcription start site (TSS) before being released into productive elongation (Henriques et al. 2013; Li et al. 2013). Since the ATPase

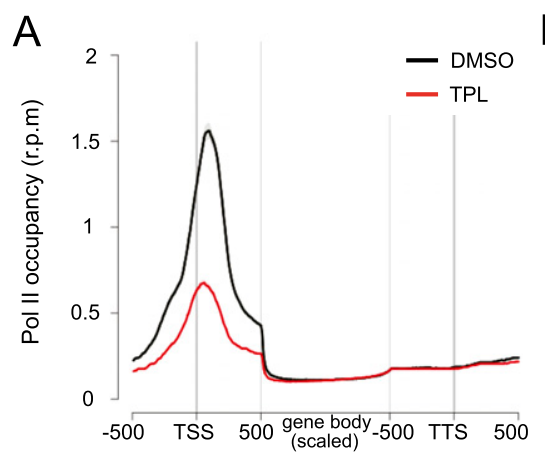

C
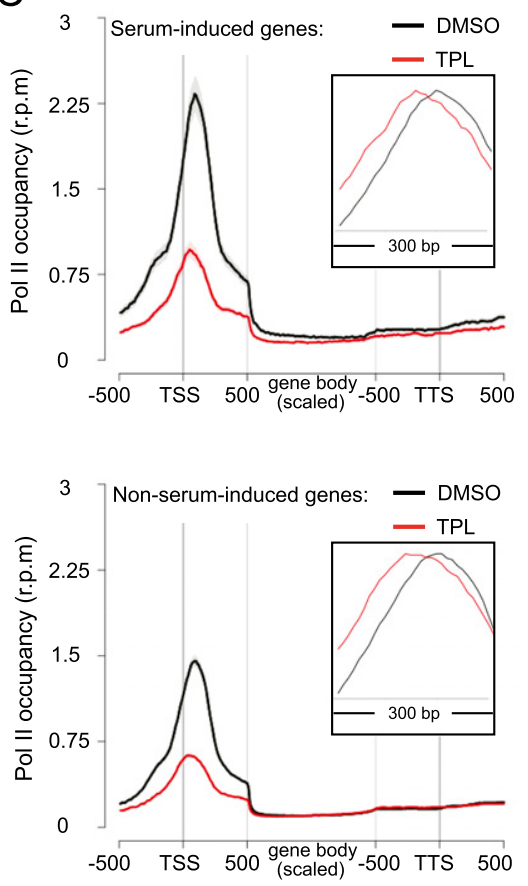

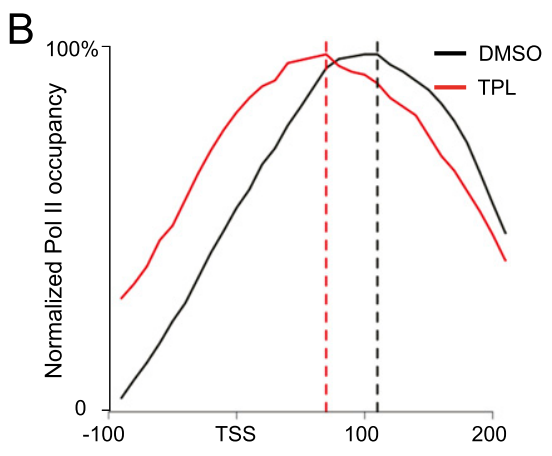

D

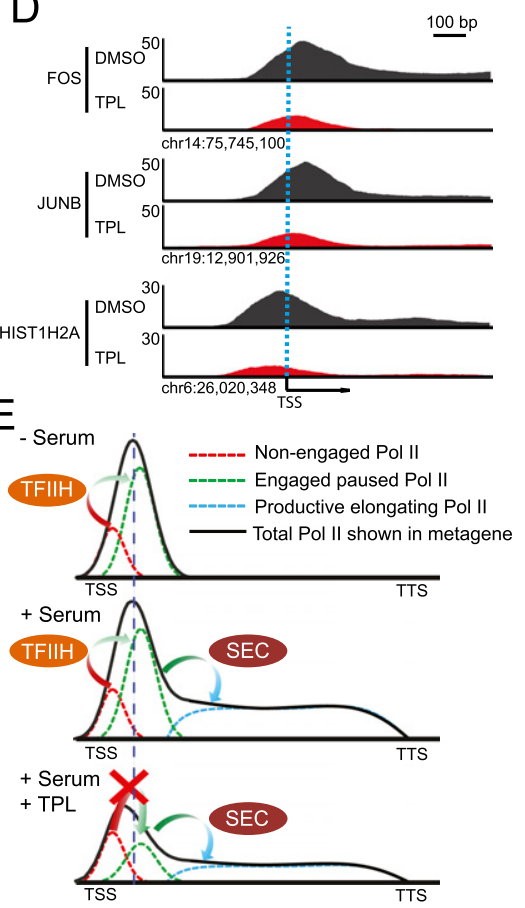

Figure 2. TPL treatment alters Pol II occupancy. (A) Metagene plots of Pol II occupancy for all genes with (red) or without (black) 60 min of TPL treatment. HCT116 cells were starved for $48 \mathrm{~h}$ before the addition of serum for $24 \mathrm{~min}$ and ChIP-seq analysis for RNA Pol II (N20 antibody). Global loss of Pol II on both promoters and gene bodies was observed as the result of TPL treatment. The shaded region indicates the $95 \%$ confidence interval of the difference between two medians. $(B)$ Metagene plot of the regions -100 base pairs (bp) to +200 bp around the TSS, plotted as the percentage of maximum for each condition. Dashed lines indicate the peak summit. In TPL-treated cells, Pol II peaks more toward the $5^{\prime}$ of the gene than in the DMSO-treated cells. $(C)$ Metagene analysis as in $A$, but with genes divided into serum-induced (top panel) and uninduced (bottom panel), is shown. The insets show the $5^{\prime}$ shift of Pol II at promoters for each gene class. $(D)$ Genome browser track examples of Pol II occupancy at promoter regions for FOS, JUNB, and HIST1H2A with (red) or without (black) TPL treatment. Dashed lines indicate the annotated TSS for each gene. $(E)$ Model for the observed shift in Pol II occupancy observed after TPL treatment. (Top panel) The promoter-bound Pol II peak is composed of nonengaged Pol II and engaged paused Pol II. The transition from the nonengaged Pol II to the engaged Pol II, transcribing $\sim 20-60 \mathrm{nt}$, requires the helicase/translocase activity of XPB/TFIIH. (Middle panel) Upon receipt of cellular signals (e.g., +serum), engaged paused Pol II is phosphorylated by P-TEFb/SEC, switches to productively elongating Pol II, and continues into gene bodies. Under normal conditions, release from the paused state allows new polymerases to enter the engaged state in a TFIIH-dependent manner. (Bottom panel)

Upon treatment of TPL, the already engaged but transiently pausing Pol II can still escape into gene bodies to complete transcription, but the transition from nonengaged Pol II to engaged Pol II is blocked, which leads to the observed decrease and 5' shift in promoterproximal Pol II occupancy. 
activity of XPB is required for promoter opening and early elongation by Pol II, the shift is consistent with TPL treatment preventing Pol II that is recruited to promoters from becoming transcriptionally engaged. Our observed 5' shift in Pol II is similar to what had been observed in the transitioning of resting to activated B cells: The peak of "loaded" Pol II in resting B cells is 5' to the peak of engaged Pol II in activated B cells (Kouzine et al. 2013).

We next separated genes based on whether they are serum-induced, as assessed by a more significant increase of Pol II occupancy after induction (Fig. 2C). Serum-induced genes have the greatest fold reduction in Pol II occupancy both at promoter-proximal regions and in the gene bodies, which is expected due to this group of genes requiring rapid rounds of initiation to reach high levels of expression within minutes (Lin et al. 2011). However, for both categories of genes, a global decrease of promoter-proximal Pol II (Fig. 2C), reduced nascent RNA (Supplemental Fig. 2B), and a 5' shift of Pol II at promoter-proximal regions are equally evident, indicating that both classes of genes require TFIIH to become transcriptionally engaged (Fig. 2C). For example, highly expressed non-serum-inducible genes such as HIST1H2A have reductions in Pol II and 5' shifts of Pol II similar to serum-inducible genes such as FOS and JUNB (Fig. 2D).

A model for the effects of TPL on Pol II is shown in Figure 2E using serum-inducible genes as examples. In the serum-starved condition, Pol II is most easily observed occupying promoter-proximal regions. However, promoter-proximal Pol II occupancy determined by ChIPseq (Fig. 2E, indicated by a black line) is a combination of engaged Pol II that is transiently pausing 20-60 nt downstream from the TSS (Fig. 2E, green dashed line) and nonengaged Pol II that has been recruited to the promoter but has not yet become transcriptionally engaged and therefore has not traveled 20-60 nt downstream (Fig. 2E, red dashed line). Upon serum stimulation, Pol II is released from the paused state into gene bodies (Fig. 2E, middle panel, blue dashed line), allowing new Pol II to be recruited. Most of this newly recruited Pol II will become transcriptionally engaged and, due to the serum-inducible conditions, is also rapidly released into gene bodies. When pretreated with TPL, only the previously engaged but pausing Pol II can be released into gene bodies, resulting in lower levels of Pol II in gene bodies (Fig. 2E, bottom panel, dashed blue line). However, as part of the response to serum, new Pol II is recruited to the promoter but, due to the presence of TPL, cannot become transcriptionally engaged and travel the 20-60 nt characteristic of transiently pausing Pol II. Thus, the average population of Pol II in the serum-induced condition (Fig. 2E, black lines in middle and bottom panels) reflects mostly transiently pausing Pol II in the absence of TPL and mostly newly recruited but nonengaged Pol II in the presence of TPL.

\section{Long residencies of paused Pol II at promoter-proximal regions}

Most genes in metazoans have an accumulation of Pol II at promoter-proximal regions due to transiently pausing Pol II (Muse et al. 2007; Adelman and Lis 2012; Grunberg and Hahn 2013). However, at a genome-wide level, it is unclear to what extent the accumulated Pol II at promoters undergoes dynamic initiation and termination or is stably tethered at promoter-proximal regions, as has been observed for Hsp70 in Drosophila (Buckley et al. 2014). To address this question, we measured the occupancy of paused Pol II before and after serum induction following TPL treatment (Fig. 3A-I). A short time of serum activation $(8 \mathrm{~min})$ was chosen to observe the earliest induction of transcription at serum-induced genes. For a small portion of serum-induced genes that are paused in the starved condition, such as FOS and ATF3, inhibition of transcription initiation by $1 \mathrm{~h}$ of TPL treatment had no detectable effect on Pol II occupancy at their promoters (Fig. 3B,D). Since TPL inhibits new Pol II from being transcriptionally engaged, the stable level of Pol II observed at FOS and ATF3 during the $1 \mathrm{~h}$ of TPL treatment indicates that the pausing Pol II is neither periodically entering into a productively elongating state nor exhibiting early termination. To determine whether these genes are TFIIH-independent (Tee et al. 2014), we measured Pol II occupancy before and after the seruminduced condition (Fig. 3, cf. B and C, and D and E). Once induced by serum, the level of Pol II increased at promoters and gene bodies for FOS and ATF3 in the absence of TPL, while, in the presence of TPL, a significant decrease of Pol II at promoters and increase at gene bodies were observed. The observation that the stably paused Pol II observed in Figure 3, B and D, in the absence of serum but in the presence of TPL can be released to its productive elongating form indicates that the stably paused Pol II was in an engaged state. However, for genes such as MYC and GAPDH, Pol II occupancy is reduced in both serum-starved and serum-activated conditions (Fig. $3 \mathrm{~F}-\mathrm{I}$ ) in the presence of TPL. Together, these data indicate that the dynamics of Pol II pausing at promoters can be highly variable (Fig. 3; Supplemental Fig. 3).

\section{Global identification and characterization of stably paused genes}

Estimates of the number of genes with paused Pol II are based on the high level of Pol II occupancy at promoters relative to gene bodies, and this measurement is referred to as the pausing index or traveling ratio (Muse et al. 2007; Zeitlinger et al. 2007; Wade and Struhl 2008; Rahl et al. 2010). It has long been known that the existence of promoter-proximal Pol II pausing is not a strict indication of expression level, with highly expressed genes such as $\beta$-actin exhibiting accumulation of Pol II at the promoter regions characteristic of pausing (Boehm et al. 2003; Adelman and Lis 2012). To distinguish between genes such as $\beta$-actin, which have very transient pausing, and genes such as Hsp70, which are mostly in a paused state until heat shock, the ratio of Pol II in the gene body to the promoter-bound Pol II has been used for genome-wide analyses (Zeitlinger et al. 2007; Rahl et al. 2010). We applied the criterion of $\log _{2}$ (body/promoter) $<4$ to define the genes with promoter-proximal paused Pol II in the starved HCT116 cells (Supplemental Fig. 4A). To determine 
A
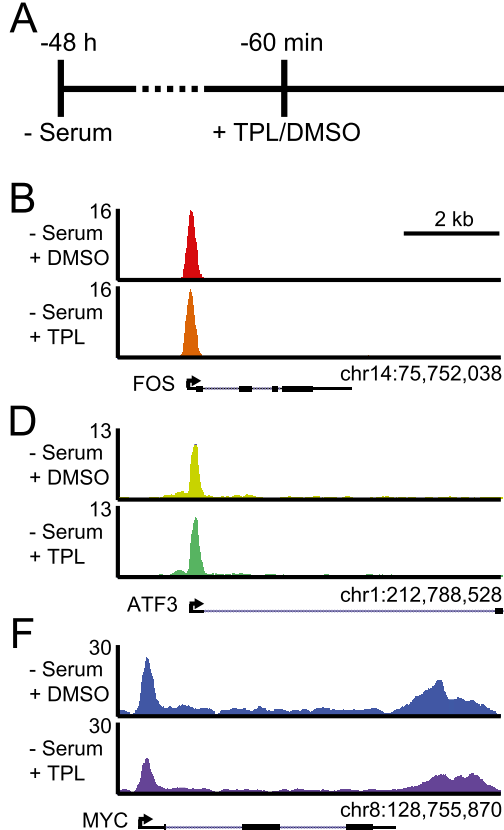

$H$

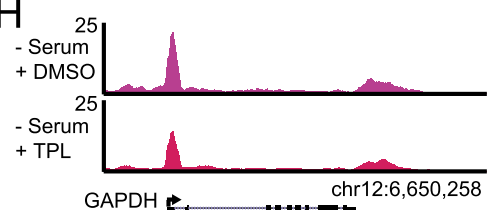

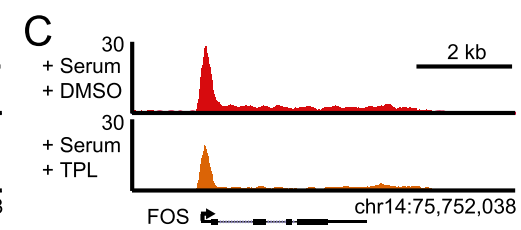
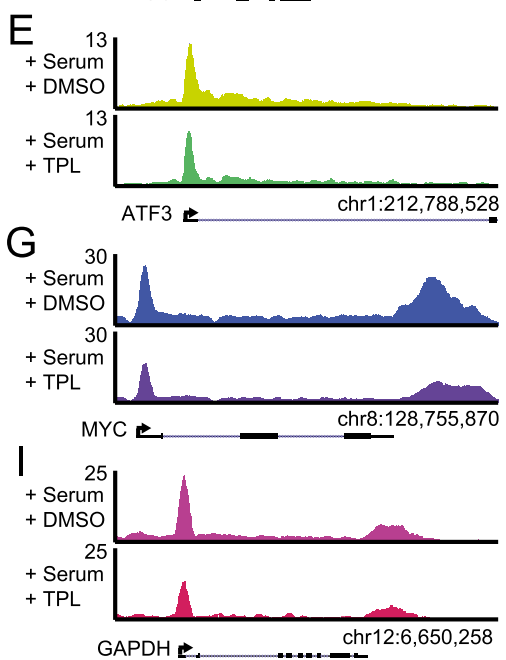

Figure 3. Different degrees of pausing. $(A-I)$ Genome browser track examples showing the contrast between stably paused Pol II and other forms of paused Pol II. (A) Schematic presentation showing the experimental design. Serum starvation began $48 \mathrm{~h}$ prior to harvesting and fixation of the cells for ChIPseq; cells were treated with TPL or the vehicle DMSO for $1 \mathrm{~h}$ prior to fixation. For serum-activated cells, serum was added 8 min prior to fixation. $(B, D, F, H)$ Genome browser track examples of Pol II occupancies in the serum-starved condition and with treatment with TPL or DMSO. $(C, E, G, I)$ Genome browser track examples of Pol II occupancies in the serum-activated condition and with treatment with TPL or DMSO.

how many genes within this large group of paused genes demonstrate the stable occupancy of Pol II comparable with FOS and ATF1, we asked which genes have a $\log _{2}$ fold change in Pol II occupancy of $<0.15$ during the $1 \mathrm{~h}$ of TPL treatment, with the $P$-value for differential occupancy being $>0.05$ (Fig. 4A). By these criteria, only $\sim 1 \%$ of genes are essentially unchanged in Pol II occupancy during $1 \mathrm{~h}$ of inhibition of initiation by TPL and demonstrate paused Pol II stability similar to what we observed for FOS and ATF1 (Fig. 4B; Supplemental Fig. 3; Supplemental Table S1).

To further characterize the genome-wide profile of this small group of stably paused genes, we performed metagene analysis comparing the stably paused genes with the other paused genes (Fig. 4C,D). The stably paused genes have occupancy of promoter-proximal Pol II comparable with that of the other paused genes. Pol II phosphorylated at Ser5 residues of the Pol II CTD (Ser5P) is a marker of promoter-proximal paused Pol II. ChIP-seq using an antibody recognizing Ser5P demonstrates that the occupancy of this form of Pol II is similar for both the stably paused and other paused genes (Supplemental Fig. 4B). However, upon TPL treatment, stably paused genes exhibit no detectable change of Pol II, while other paused genes show a significant loss of Pol II at promoter-proximal regions (Fig. 4C,D).

The difference in changes in occupancy of stably paused and other paused genes at promoter-proximal regions could be due to the paused Pol II at promoters of other paused genes either periodically entering gene bodies to finish transcription during the $1 \mathrm{~h}$ of TPL treatment or terminating prematurely. To distinguish between these possibilities, we performed nascent RNAseq in the starved HCT116 cells. We observed that the stably paused genes have significantly less expression than other paused genes, suggesting that stably paused genes could be distinguished by a less frequent release of Pol II into gene bodies (Fig. 4E).

In principle, the formation of promoter-proximal paused Pol II is composed of at least three steps: generating newly engaged paused Pol II that synthesizes short RNAs, restraining early termination after transcription initiation, and preventing productive elongation. The identification of stably paused Pol II through inhibition of TFIIH raises the question of the nature of its regulation as compared with the class of other paused genes that do not seem to be as stable in their pausing state. NELF is well studied for its role in the formation of paused Pol II (Yamaguchi et al. 1999; Henriques et al. 2013). To answer this question, we depleted NELF-A in the serum-starved condition and performed Pol II ChIP-seq. Metagene analysis showed that changes in Pol II occupancy at promoters is negligible for stably paused genes and significantly decreased for other paused genes (Fig. 4F, G). The reduced promoter-proximal Pol II occupancy at paused genes that are not stably paused is consistent with the proposed role for NELF in maintaining general pausing (Wu et al. 2003; Gilchrist et al. 2010). On the contrary, stably paused genes are less dependent on NELF for the maintenance of pausing. We also measured the 
A
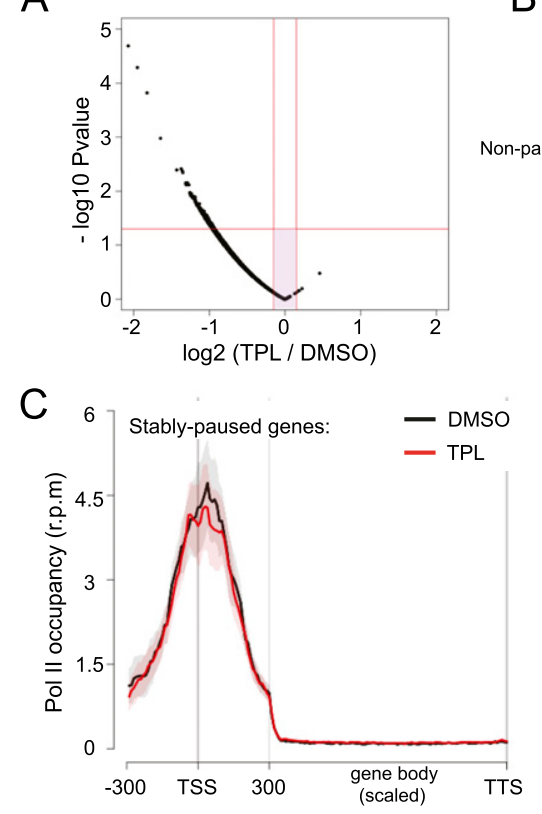

$\mathrm{F}$

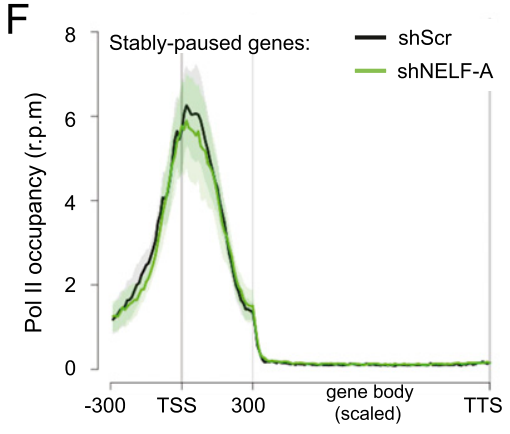

B

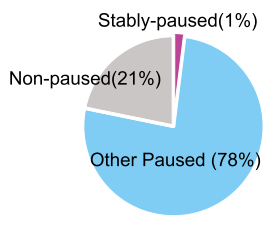

$\mathrm{E}$
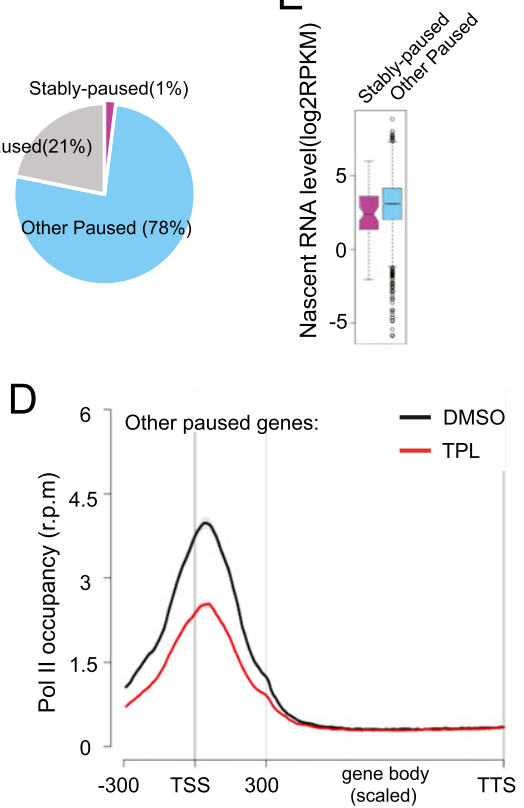

G

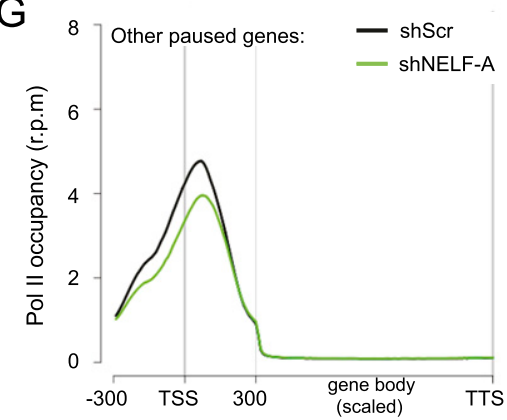

Figure 4. Global identification and characterization of stably paused genes. (A) Volcano plot representing the criteria for the classification of Pol II on stably paused genes. Stably paused genes, highlighted in pink, are the subset of paused genes with a $\log _{2}$ fold change in Pol II occupancy during $1 \mathrm{~h}$ of TPL treatment of $<0.15$ and a $P$-value for differential occupancy of $>0.05$ $\left(-\log _{10} P\right.$-value $\left.<1.3\right)$. (B) Pie chart representing the percentage of stably paused genes, other forms of paused genes, and nonpaused genes. $(C$, D) Metagene analysis of Pol II occupancy for stably paused genes with (red) or without (black) TPL treatment. (E) Box plot analysis of nascent RNA levels for stably paused genes and other forms of paused genes, confirming that stably paused genes release less productively elongating Pol II than the majority of genes exhibiting paused Pol II. $(F, G)$ Metagene analysis of Pol II occupancy for stably paused genes and other forms of paused genes targeted by a scrambled shRNA (shScr; black) or NELF-A shRNA (shNELF-A; lime). expression of stably paused genes by real-time PCR for comparison. Interestingly, the stably paused genes displayed an increase in expression in NELF knockdown cells (Supplemental Fig. 4D), indicating a limited increase in Pol II escape into gene bodies. However, this level of escape must be extremely infrequent in the population of cells, since reduction of Pol II was not observed during $1 \mathrm{~h}$ of TPL treatment. Thus, we conclude that NELF is more important in the maintenance of pausing for nonstably paused genes compared with stably paused genes, indicating that other factors are responsible for stable pausing. However, we note that it is possible that Pol II at stably paused genes has a stronger affinity for NELF than Pol II on other genes, and therefore RNAi of NELF could result in the preferential association of residual NELF with these genes. To distinguish between these possibilities will require repeating these analyses in NELF knockout cells.

\section{Discussion}

Our genome-wide gene expression studies in cells treated with TPL and cells treated with shRNA toward the subunits of TFIIH (XPB) suggest that XPB is a major target of TPL in regards to transcriptional regulation (Supplemental Fig 1). Furthermore, our results demonstrate that the lack of change in Pol II occupancy during TPL treatment for promoters of genes bearing stably paused Pol II is not the result of these genes being independent of TFIIH activity, as reported for some Erk2-regulated genes (Tee et al. 2014). In addition, the long residency of Pol II indicates that these genes are not undergoing frequent rounds of initiation and early termination, as seen at some genes with high levels of promoter-proximal Pol II (Brannan et al. 2012; Wagschal et al. 2012).

Using TPL as a XPB-specific inhibitor, our genomewide studies have provided insight into the dynamics of promoter-proximal Pol II. Our genome-wide analysis revealed the existence of a small group of genes with paused Pol II stably tethered to the promoter, which we call stably paused genes. For this group of genes, the inhibition of transcription initiation by TPL does not lead to changes in Pol II occupancy or expression during the $1 \mathrm{~h}$ of treatment, illuminating the steady nature of this paused Pol II state. For serum-induced genes within this group, serum activation leads to a dramatic increase of Pol II occupancy and an up-regulation of expression. This induction is inhibited by TPL treatment (Figs. 1, 3). 
However, the serum-induced genes that are stably paused are still capable of entering the productive transcription state following induction while under the TPL treatment (Fig. 3). This observation indicates that promoter-bound Pol IIs that are stably paused are fully capable of transcriptional engagement.

NELF is the most widely studied factor promoting pausing (Yamaguchi et al. 2013). We found that while NELF is important in the maintenance of pausing for nonstably paused genes, other yet-to-be-determined factors could regulate the maintenance of pausing at stably paused genes. Such factors could include Gdown1, which has emerged as a factor that promotes Pol II pausing through the inhibition of early termination by TTF2 and by preventing the binding of the early elongation factor TFIIF (Cheng et al. 2012; Espinosa 2012; Jishage et al. 2012).

It will be very interesting to mechanistically define what the molecular differences are between Pol II that is stably paused at some promoters compared with paused Pol II with a low residence time at other genes. Our studies recently demonstrated that the binding of transcription factors such as ELL3 at enhancers is involved in setting up Pol II pausing at promoters and that the interactions between ELL3 at enhancers and the SEC at promoters could regulate transcriptional induction and pause/release (Lin et al. 2013). Therefore, it would be interesting to define the enhancer status, its chromatin modification patterns, and transcription factor interactions between stably paused genes and genes with paused Pol II with a low residence time. We also do not know the level of the SEC and the activity of CDK9 within the SEC on these two classes of genes. It will also be very illuminating to study the three-dimensional interactions and localization patterns of genes with different forms of paused Pol II.

In the final analysis, the use of TPL as a TFIIH-specific inhibitor, the identification of different forms of paused Pol II with varying residence times at the promoters of different genes, and the identification of stably paused genes could pave the way for evaluating how the various pausing/elongation factors, chromatin-modifying machineries, and cis-regulatory elements function to establish and regulate pausing in independent and cooperative manners to regulate developmental processes in metazoans. Given the fact that the misregulation of the elongation stage of transcription has been associated with human diseases, including cancer (Smith and Shilatifard 2013), the use of TPL for the in vivo study of transcriptional elongation control should be very informative.

\section{Materials and methods}

\section{Antibodies and cell lines}

Human Pol II (N-20) monoclonal antibody was purchased from Santa Cruz Biotechnology. 8WG16 anti-CTD (MMS-126R), H5 Ser2P-CTD (MMS-129R), and H14 Ser5P-CTD (MMS-134R) monoclonal antibodies were purchased from Covance. 3E8 Ser5PCTD monoclonal antibodies were purchased from Millipore. TFIIB (C-18) and XPB (S-19) antibodies were purchased from Santa Cruz Biotechnology. AFF4 and CDK9 antibodies were made in-house. Anti- $\beta$-tubulin E7 monoclonal antibody was purchased from the Developmental Hybridoma Studies Bank. HCT116 cells were grown in DMEM supplemented with 10\% FBS.

\section{ChIP-seq}

For each ChIP assay, $5 \times 10^{7}$ cells were used, as described (Lee et al. 2006). Briefly, HCT116 cells were cross-linked with $1 \%$ paraformaldehyde for $10 \mathrm{~min}$ at room temperature with gentle rotation and then quenched with $0.125 \mathrm{M}$ glycine. After washing, nuclei were sonicated on a Misonix Sonicator 3000 ultrasonic cell disruptor, and the supernatant was used for immunoprecipitation with the indicated antibody. ChIP-seq libraries were prepared with Illumina's TruSeq DNA sample preparation kit.

Sequencing data were acquired through the default Illumina pipeline using Casava version 1.8. Reads were aligned to the human genome (University of California at Santa Cruz [UCSC] hg19) using the Bowtie aligner version 0.12.9, allowing uniquely mapping reads only and allowing up to two mismatches (Langmead et al. 2009). Reads were extended to 150 bases toward the interior of the sequenced fragment and normalized to total reads aligned (reads per million [RPM]). Gene annotations and TSS information were from Ensembl 67 using only RefSeq mRNA entries.

The R package edge R 3.0.8 was used to perform differential ChIP-seq analysis (Robinson et al. 2010). In the serum-induced condition, the serum-induced genes were the genes where Pol II in the gene body increased significantly $(P$-value $<0.05)$ when comparing $24 \mathrm{~min}$ and $0 \mathrm{~min}$ of serum treatment. Genes with Pol II coverage $>0.5$ RPKM (reads per million in the gene body divided by gene length and multiplied by $1 \mathrm{~kb}$ ) in the 24-min serum treatment were used in the metagene plots. In the serumstarved condition, -100 base pairs (bp) to $200 \mathrm{bp}$ of the TSS was used as the promoter region of each gene. The genes that were highly enriched with Pol II in the serum-starved condition were selected for the pausing analysis in which the reads were $>5$ RPM at the promoter region in each replicate. Two biological replicates were used. The pausing genes were those genes with a $\log _{2}$ body/promoter ratio of Pol II that was less than -4 . The stably pausing genes were the pausing genes with the absolute value of $\log _{2}$ fold change of Pol II in the promoter regions that was $<0.15$ and $P$-value was $>0.05$.

The metagene plots were made by the median Pol II occupancy of multiple genes. The shadow region marked an $\sim 95 \%$ confidence interval for the difference in two medians, which is between the lower and upper extremes of the "notch" $[ \pm 1.58$ $\mathrm{IQR} / \mathrm{sqrt}(\mathrm{n})]$ in box plots. Gene body regions that were marked as "scaled gene body" were scaled into the same 1000-bp length. All of the metagene plots were binned into $10 \mathrm{bp}$. The shift of Pol II was the distance between two maximum points of the medians.

\section{Nascent RNA-seq}

The nascent RNA-seq protocol was adapted from a previously described method (Khodor et al. 2011). Briefly, $1 \times 10^{8}$ HCT116 cells were harvested and washed three times with cold PBS and then suspended in $10 \mathrm{~mL}$ of buffer A (10 mM HEPES at $\mathrm{pH} 7.9,10$ $\mathrm{mM} \mathrm{KCl}, 2 \mathrm{mM} \mathrm{MgCl} 2,1 \mathrm{mM}$ DTT, $1 \times$ Complete protease inhibitors [Roche]). After incubating for $15 \mathrm{~min}$ on ice, the cells were homogenized in a precooled $15-\mathrm{mL}$ Dounce tissue homogenizer 15 times. Nuclei were then washed twice with buffer B (10 mM HEPES at pH 7.9, $250 \mathrm{mM}$ sucrose, $1 \mathrm{mM}$ DTT, $1 \times$ complete protease inhibitors). The pellet was vigorously suspended with $1 \mathrm{~mL}$ of NUN buffer (20 mM HEPES at $\mathrm{pH} 7.9,7.5 \mathrm{mM}$ $\mathrm{MgCl}_{2}, 0.2 \mathrm{mM}$ EDTA, $300 \mathrm{mM} \mathrm{NaCl}, 1 \mathrm{M}$ urea, $1 \%$ [v/v] Nonidet P40, $1 \mathrm{mM}$ DTT, $20 \mathrm{U} / \mathrm{mL}$ SUPERase.In RNase in- 
hibitor [Ambion]) that was freshly prepared. The chromatin was then washed twice more with $5 \mathrm{~mL}$ of NUN buffer each time. The supernatant was removed, and TRIzol reagent (Invitrogen) was added to the pellet for purification of RNA. The RNA was subjected to polyA depletion with Oligo(dT) magnetic beads (Invitrogen) and DNase I treatment (New England Biolabs) for 20 min and then repurified. For sequencing, $2 \mu \mathrm{g}$ of resulting RNA was used for ribosomal RNA depletion with the RiboZero kit (Epicenter), and libraries were made with the TruSeq RNA sample preparation kit (Illumina).

Three biological replicates were used in the analysis for comparing the transcription levels of stably paused and other paused genes. Sequencing data were acquired through the default Illumina pipeline using Casava version 1.8. Reads were aligned to the human genome UCSC hg19 and gene annotations from Ensembl 67 using TopHat version 2.0.9 using option -g 1 and allowing up to two mismatches.

\section{Lentivirus-mediated RNAi}

For nascent RNA-seq in the normal serum condition, HCT116 cells were infected with lentivirus containing either a scrambled control shRNA (shScr) or XPB shRNA (shXPB; clone ID: TRCN0000022081) in the presence of $8 \mu \mathrm{g} / \mathrm{mL}$ Polybrene (Sigma) for $24 \mathrm{~h}$ in DMEM supplemented with $10 \%$ FBS. The infected cells were selected with $2 \mu \mathrm{g} / \mathrm{mL}$ puromycin for an extra $48 \mathrm{~h}$ before harvest. For ChIP-seq in the starved condition, HCT116 cells were infected with lentivirus containing either a scrambled control shRNA (shScr) or NELF-A shRNA (shNELF-A; clone ID: TRCN0000149873) in the presence of $8 \mu \mathrm{g} / \mathrm{mL}$ Polybrene (Sigma) for $24 \mathrm{~h}$ in DMEM supplemented with $10 \%$ FBS. The infected cells were selected with $2 \mu \mathrm{g} / \mathrm{mL}$ puromycin for an extra $48 \mathrm{~h}$ before harvest. The cells were also starved for $24 \mathrm{~h}$ before $1 \mathrm{~h}$ of TPL/DMSO treatment and harvest.

\section{Acknowledgments}

We thank Dr. Edwin Smith for conversations throughout this study and throughout the writing of the manuscript, Dr. Julia Zeitlinger for critical reading of the manuscript, Malcolm Cook for help with data analysis, the Molecular Biology core facility at Stowers Institute for creating and sequencing libraries for next-generation sequencing, and the Tissue Culture core facility for large-scale cell culture. These studies were supported in part by National Institutes of Health grant R01GM069905 to A.S.

\section{References}

Adelman K, Lis JT. 2012. Promoter-proximal pausing of RNA polymerase II: emerging roles in metazoans. Nat Rev Genet 13: $720-731$.

Boehm AK, Saunders A, Werner J, Lis JT. 2003. Transcription factor and polymerase recruitment, modification, and movement on dhsp70 in vivo in the minutes following heat shock. Mol Cell Biol 23: 7628-7637.

Brannan K, Kim H, Erickson B, Glover-Cutter K, Kim S, Fong N, Kiemele L, Hansen K, Davis R, Lykke-Andersen J, et al. 2012. mRNA decapping factors and the exonuclease Xrn2 function in widespread premature termination of RNA polymerase II transcription. Mol Cell 46: 311-324.

Buckley MS, Kwak H, Zipfel WR, Lis JT. 2014. Kinetics of promoter Pol II on Hsp70 reveal stable pausing and key insights into its regulation. Genes Dev 28: 14-19.

Cheng B, Li T, Rahl PB, Adamson TE, Loudas NB, Guo J, Varzavand K, Cooper JJ, Hu X, Gnatt A, et al. 2012.
Functional association of Gdown1 with RNA polymerase II poised on human genes. Mol Cell 45: 38-50.

Core LJ, Lis JT. 2008. Transcription regulation through promoter-proximal pausing of RNA polymerase II. Science 319: 1791-1792.

Espinosa JM. 2012. Get back TFIIF, don't let me Gdown1. Mol Cell 45: 3-5.

Gilchrist DA, Dos Santos G, Fargo DC, Xie B, Gao Y, Li L, Adelman K. 2010. Pausing of RNA polymerase II disrupts DNA-specified nucleosome organization to enable precise gene regulation. Cell 143: 540-551.

Goodrich JA, Tjian R. 1994. Transcription factors IIE and IIH and ATP hydrolysis direct promoter clearance by RNA polymerase II. Cell 77: 145-156.

Goodrich JA, Cutler G, Tjian R. 1996. Contacts in context: promoter specificity and macromolecular interactions in transcription. Cell 84: 825-830.

Grunberg S, Hahn S. 2013. Structural insights into transcription initiation by RNA polymerase II. Trends Biochem Sci 38: 603-611.

Guenther MG, Levine SS, Boyer LA, Jaenisch R, Young RA. 2007. A chromatin landmark and transcription initiation at most promoters in human cells. Cell 130: 77-88.

Henriques T, Gilchrist DA, Nechaev S, Bern M, Muse GW, Burkholder A, Fargo DC, Adelman K. 2013. Stable pausing by RNA polymerase II provides an opportunity to target and integrate regulatory signals. Mol Cell 52: 517-528.

Hsin JP, Manley JL. 2012. The RNA polymerase II CTD coordinates transcription and RNA processing. Genes Dev 26: 2119-2137.

Jishage M, Malik S, Wagner U, Uberheide B, Ishihama Y, Hu X, Chait BT, Gnatt A, Ren B, Roeder RG. 2012. Transcriptional regulation by $\mathrm{Pol} \mathrm{II}(\mathrm{G})$ involving mediator and competitive interactions of Gdownl and TFIIF with Pol II. Mol Cell 45: 51-63.

Jonkers I, Kwak H, Lis JT. 2014. Genome-wide dynamics of Pol II elongation and its interplay with promoter proximal pausing, chromatin, and exons. eLife 3: e02407.

Khodor YL, Rodriguez J, Abruzzi KC, Tang CH, Marr MT 2nd, Rosbash M. 2011. Nascent-seq indicates widespread cotranscriptional pre-mRNA splicing in Drosophila. Genes Dev 25: 2502-2512.

Kouzine F, Wojtowicz D, Yamane A, Resch W, Kieffer-Kwon KR, Bandle R, Nelson S, Nakahashi H, Awasthi P, Feigenbaum L, et al. 2013. Global regulation of promoter melting in naive lymphocytes. Cell 153: 988-999.

Kwak H, Lis JT. 2013. Control of transcriptional elongation. Annu Rev Genet 47: 483-508.

Lagha M, Bothma JP, Esposito E, Ng S, Stefanik L, Tsui C, Johnston J, Chen K, Gilmour DS, Zeitlinger J, et al. 2013. Paused Pol II coordinates tissue morphogenesis in the Drosophila embryo. Cell 153: 976-987.

Langmead B, Trapnell C, Pop M, Salzberg SL. 2009. Ultrafast and memory-efficient alignment of short DNA sequences to the human genome. Genome Biol 10: R25.

Lee TI, Johnstone SE, Young RA. 2006. Chromatin immunoprecipitation and microarray-based analysis of protein location. Nat Protoc 1: 729-748.

Li J, Liu Y, Rhee HS, Ghosh SK, Bai L, Pugh BF, Gilmour DS. 2013. Kinetic competition between elongation rate and binding of NELF controls promoter-proximal pausing. Mol Cell 50: 711-722.

Lin C, Garrett AS, De Kumar B, Smith ER, Gogol M, Seidel C, Krumlauf R, Shilatifard A. 2011. Dynamic transcriptional events in embryonic stem cells mediated by the super elongation complex (SEC). Genes Dev 25: 1486-1498. 
Lin C, Garruss AS, Luo Z, Guo F, Shilatifard A. 2013. The RNA Pol II elongation factor Ell3 marks enhancers in ES cells and primes future gene activation. Cell 152: 144-156.

Luo Z, Lin C, Guest E, Garrett AS, Mohaghegh N, Swanson S, Marshall S, Florens L, Washburn MP, Shilatifard A. 2012a. The super elongation complex family of RNA polymerase II elongation factors: gene target specificity and transcriptional output. Mol Cell Biol 32: 2608-2617.

Luo Z, Lin C, Shilatifard A. 2012b. The super elongation complex (SEC) family in transcriptional control. Nat Rev Mol Cell Biol 13: 543-547.

Manzo SG, Zhou ZL, Wang YQ, Marinello J, He JX, Li YC, Ding J, Capranico G, Miao ZH. 2012. Natural product triptolide mediates cancer cell death by triggering CDK7-dependent degradation of RNA polymerase II. Cancer Res 72: 5363-5373.

Miki TS, Grosshans H. 2013. The multifunctional RNase XRN2. Biochem Soc Trans 41: 825-830.

Muse GW, Gilchrist DA, Nechaev S, Shah R, Parker JS, Grissom SF, Zeitlinger J, Adelman K. 2007. RNA polymerase is poised for activation across the genome. Nat Genet 39: 1507-1511.

Rahl PB, Lin CY, Seila AC, Flynn RA, McCuine S, Burge CB, Sharp PA, Young RA. 2010. c-Myc regulates transcriptional pause release. Cell 141: 432-445.

Rasmussen EB, Lis JT. 1993. In vivo transcriptional pausing and cap formation on three Drosophila heat shock genes. Proc Natl Acad Sci 90: 7923-7927.

Robinson MD, McCarthy DJ, Smyth GK. 2010. edgeR: a Bioconductor package for differential expression analysis of digital gene expression data. Bioinformatics 26: 139-140.

Smith E, Shilatifard A. 2013. Transcriptional elongation checkpoint control in development and disease. Genes Dev 27: 1079-1088.

Tee WW, Shen SS, Oksuz O, Narendra V, Reinberg D. 2014. Erk1/2 activity promotes chromatin features and RNAPII phosphorylation at developmental promoters in mouse ESCs. Cell 156: 678-690.

Titov DV, Gilman B, He QL, Bhat S, Low WK, Dang Y, Smeaton M, Demain AL, Miller PS, Kugel JF, et al. 2011. XPB, a subunit of TFIIH, is a target of the natural product triptolide. Nat Chem Biol 7: 182-188.

Wade JT, Struhl K. 2008. The transition from transcriptional initiation to elongation. Curr Opin Genet Dev 18: 130-136.

Wagschal A, Rousset E, Basavarajaiah P, Contreras X, Harwig A, Laurent-Chabalier S, Nakamura $\mathrm{M}$, Chen $\mathrm{X}$, Zhang $\mathrm{K}$, Meziane O, et al. 2012. Microprocessor, Setx, Xrn2, and Rrp6 co-operate to induce premature termination of transcription by RNAPII. Cell 150: 1147-1157.

Wang Y, Lu JJ, He L, Yu Q. 2011. Triptolide (TPL) inhibits global transcription by inducing proteasome-dependent degradation of RNA polymerase II (Pol II). PLoS ONE 6: e23993.

Wu CH, Yamaguchi Y, Benjamin LR, Horvat-Gordon M, Washinsky J, Enerly E, Larsson J, Lambertsson A, Handa H, Gilmour D. 2003. NELF and DSIF cause promoter proximal pausing on the hsp70 promoter in Drosophila. Genes Dev 17: 1402-1414.

Yamaguchi Y, Takagi T, Wada T, Yano K, Furuya A, Sugimoto S, Hasegawa J, Handa H. 1999. NELF, a multisubunit complex containing RD, cooperates with DSIF to repress RNA polymerase II elongation. Cell 97: 41-51.

Yamaguchi Y, Shibata H, Handa H. 2013. Transcription elongation factors DSIF and NELF: promoter-proximal pausing and beyond. Biochim Biophys Acta 1829: 98-104.

Zeitlinger J, Stark A, Kellis M, Hong JW, Nechaev S, Adelman K, Levine M, Young RA. 2007. RNA polymerase stalling at developmental control genes in the Drosophila melanogaster embryo. Nat Genet 39: 1512-1516. 


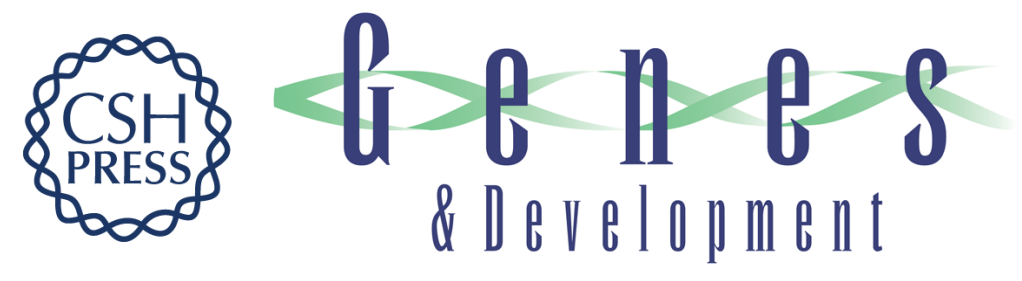

\section{Stably paused genes revealed through inhibition of transcription initiation by the TFIIH inhibitor triptolide}

Fei Chen, Xin Gao and Ali Shilatifard

Genes Dev. 2015, 29:

Access the most recent version at doi:10.1101/gad.246173.114

Supplemental
Material http://genesdev.cshlp.org/content/suppl/2014/12/29/29.1.39.DC1

References This article cites 42 articles, 11 of which can be accessed free at: http://genesdev.cshlp.org/content/29/1/39.full.html\#ref-list-1

Creative This article is distributed exclusively by Cold Spring Harbor Laboratory Press for the first Commons License

Email Alerting Service six months after the full-issue publication date (see http://genesdev.cshlp.org/site/misc/terms.xhtml). After six months, it is available under a Creative Commons License (Attribution-NonCommercial 4.0 International), as described at http://creativecommons.org/licenses/by-nc/4.0/.

Receive free email alerts when new articles cite this article - sign up in the box at the top right corner of the article or click here.

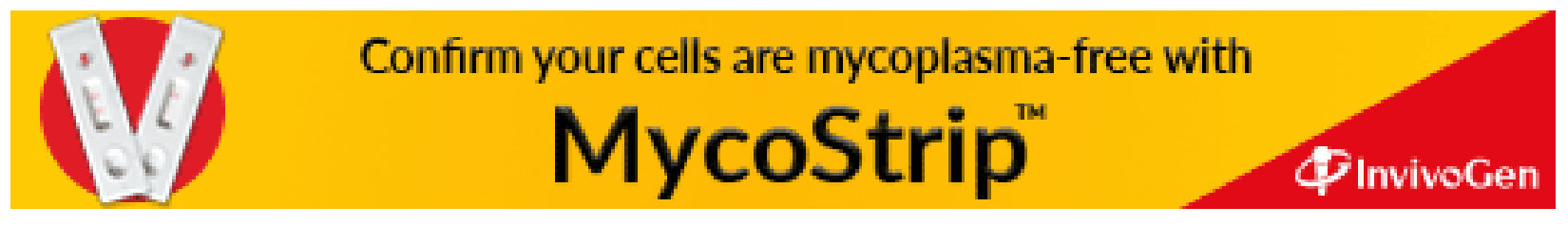

